\title{
UTILIZAÇÃO DO FILTRO HODRICK-PRESCOTT NAS ANÁLISES DE DADOS CLIMÁTICOS
}

\author{
SIQUEIRA, Anderlan Henrique Batista - anderlansiqueira@gmail.com \\ MOLION, Luiz Carlos Baldicero - Icmolion@gmail.com \\ Instituto de Ciências Atmosféricas, Universidade Federal de Alagoas.
}

\begin{abstract}
RESUMO: Este estudo teve como objetivo introduzir e demonstrar a utilização do filtro HodrickPrescott na análise e decomposição de séries temporais meteorológicas de longo prazo. Fez-se um exercício comparativo entre a técnica estatística do filtro Hodrick-Prescott e a das Médias Móveis, com um parâmetro de filtro de 13 meses, a fim de avaliar o desempenho de ambas na decomposição de altas e baixas frequências, ou seja, variabilidade climática de curtos e longos períodos, respectivamente. Como exemplo prático, aplicaram-se as duas técnicas na série do índice da Oscilação Decadal do Pacifico (ODP) para separar a variabilidade decadal (tendência) da interanual (partecíclica/aleatória). Utilizou-se a análise das ondeletas de Morlet nas séries da tendência e parte cíclica/aleatória, geradas com as duas técnicas, para identificar os padrões oscilatórios existentes e suas respectivas periodicidades aparentes. As técnicas conseguiram representar bem as oscilações decadal e interanual. Contudo, o filtro Hodrick-Prescott demonstrou ser uma técnica mais adequada, pois removeu efetivamente as frequências altas da série original, produzindo uma curva de tendência suave, e as incorporou na curva cíclica/aleatória. Esse fato foi confirmado nas análises da ondeletas das duas decomposições, tendência e parte cíclica/aleatória. O filtro Hodrick-Prescott apresenta, ainda, uma grande vantagem sobre a técnica das médias móveis, já que não há perda de dados no início e no final da série. Quanto à interpretação física dos resultados foram confirmados o ciclo longo de 50-60 anos da ODP e os ciclos curtos de 3 a 7 anos, relativos aos eventos ENOS já encontrado na literatura.
\end{abstract}

PALAVRAS-CHAVES:Análise de séries temporais, tendência climática, ondeletas.

USE OF THE HODRICK-PRESCOTT FILTER TO CLIMATE DATA ANALYSIS

ABSTRACT: This study aimed at introducingand demonstrating the use of the Hodrick-Prescott filter in the analysis of climate data time series. A comparison was made between the techniques Hodrick - Prescott filter and the Moving Averages, with a filter parameter of 13 months, in order to evaluate the performance of both techniques regarding the decomposition of the high and low frequencies contained in the original time series, respectively short and long term climate variability. As a practical example, both techniques were applied to the Pacific Decadal Oscillation (PDO)Index time series to separate decadal variability (trend) fromthe interannual variability (cyclic/random part). The Morlet wavelet analysis was applied to the trend and cyclic/random series generated with the two techniques to check existence of oscillatory patterns and their apparent periodicities. Both techniques representedwell the decadal and the interannual climate variability. However, the Hodrick-Prescott filter proved to be a more adequatetechnique, since it removed the high frequencies of the original time series efficiently, resulting in a smooth trend curve, and incorporated the high frequencies into the cyclic/random curve.This was confirmed with the wavelet analysis of the two decompositions, trend and cyclic/random series. In addition, the Hodrick-Prescott filter has a great advantage when compared to the Moving Averages techniques because there is no loss of data at the beginning and at the end of the time series. The physical interpretation of the results confirmed the PDO 50-60 year cycle and the interannual 3 to7 year cycles, associated with ENSO events, already described in the literature.

KEY-WORDS: Time series analysis, climate trend, wavelets.

\section{Introdução}

Uma série temporal é definida como um conjunto de observação de uma determinada variável em relação ao tempo. Geralmente, esses intervalos de tempo são iguais. No lado matemático, uma série temporal é demonstrada pela relação entre uma variável " $Y$ " qualquer e o seu comportamento no tempo ( $t)$. Portanto, a variável $Y$ está em função de $t$, isto é, $Y=f(t)$. Essa função pode ser representada graficamente pela construção de um gráfico de $Y$ pelo tempo $(t)$, por exemplo, um gráfico da variação climática da Oscilação Decadal do Pacífico (ODP) que é a variação da temperatura da superfície do mar (TSM) no Oceano 
Pacifico durante um determinado período, entre 25 e 30 anos cada fase, totalizando um ciclo de 50 a 60 anos (MANTUA et al., 1997).Observou-se queuma série temporal é formada por componentes características (SPIEGEL, 1969). A análise dessas componentes tem grande valor para prognósticosclimáticos.

Essas componentes devem ser classificadas para estudos. Tal classificação também é conhecida como análise das componentes da série temporal. As componentes podem ser divididas em quatro partes essenciais: sinais ou variabilidades de longo prazo de tempo, de curto prazo, cíclicas e aleatórias. A variabilidade de longo prazo de tempoé caracterizada por oscilações quase seculares e/ou interdecadais. Nesses sinais, observa-se que a série temporal, aparentemente, se desenvolve em uma direção geral, retaou curvas sinuosas, em intervalos de tempo longo, definindo a curva de tendência. Os sinais cíclicos representam oscilações ou desvios de prazo interanual ou plurianual, existentes na curva da tendência geral da série. As oscilações em escala de curto prazo de tempo referem-se aos padrões existentes, idênticos ou quase idênticos, que são observados na escala anual e/ou sazonal da série. Finalmente, oscilações aleatórias (irregulares) estão associadas aos deslocamentos esporádicos que são provocados por eventos casuais, como, por exemplo, o impacto de uma erupção vulcânica na temperatura da superfície do mar. A separação dessas 4 componentes auxilia no entendimento dos processos físicos, sociais e antrópicos, e suas escalas de tempo, atuantes sobre uma dada variável.

Quando se trata de flutuações climáticas propriamente ditas, observa-se que as escalas de tempo e o comportamento das variáveis climáticas condicionam a frequência e intensidade de alguns fenômenos. Um exemplo foi o demonstrado por Molion (2005) que descreveu uma inesperada coincidência entre o comportamento da temperatura do ar média global (Ta) e a ODP, em que a Ta diminuiu entre os anos de 1947 - 1976 (fase fria da ODP) e aumentou durante 1976 - 1998 (fase quente da ODP). Observou também que, durante a fase fria da ODP, os eventos La Niña foram mais intensos, longos e mais frequentes e os fenômenos El Niños foram menos intensos, curtos e menos frequentes. Na fase quente da ODP, ocorreu o oposto. Então, ficou evidenciado que a ODP influenciou os eventos El Niño - Oscilação Sul (ENOS) e que, por sua vez, influenciaram a Ta. Ou seja, durante um evento El Niño (La Niña), a Ta tende a ficar acima (abaixo) da normal climática provocando um aquecimento (resfriamento) global. Alguns autores (RASMUSSON e WALLACE, 1983; ROPELEWSKI e HALPERT, 1987; NOBRE e MOLION, 1988; MOLION, 2005) já haviam reportado esse impacto global.

A separação das 4 componentes características de uma série não é uma tarefa simples. Uma das técnicas mais utilizada, e questionável, é a das Médias Móveis,conhecida também como regularização das series temporais. Porém, existem algumas limitações nessa técnica, uma delas é que se podem gerar movimentos cíclicos ou de natureza espúria que não existem nos dados originais. Baseado nessas informações, o presente artigo tem como objetivo 
introduzir uma nova técnica estatística muito utilizada em Economia. Essa técnica foi empregada para a análise das series temporais de dados meteorológicos/climáticose é conhecida por Filtro Hodrick-Prescott (FHP), desenvolvido por Hodrick e Prescott (1980). Especificamente, estudou-se a adequação dessa técnica na separação das componentes de longo prazo, de curto prazoe aleatórias na série temporal do índice da ODP, em particular, se as componentes das novas series geradas mantêm os mesmo padrões da série original. Também foi feito um estudo comparativo entre o FHP e a técnica das Médias Móveis.

\section{Materiais e métodos}

\subsection{Dados e programas utilizados}

O conjunto de dados utilizados para esse estudo foi a série temporaldosíndices deOscilação Decadal do Pacifico (IODP), que dizem respeito à variabilidade da temperatura da superfície do mar (TSM) nesse oceano. Os dados cobrem o período de janeiro de 1900 a dezembro de 2011. O índice da ODP foi coletado no site do Earth System ResearchLaboratory (ESRL/PSD/NOAA). No primeiro momento, aplicou-se o filtro Hodrick-Prescott (FHP) nas series dos índices da ODP para separar a tendência (variabilidade de longo prazo) da série de sua parte cíclica/aleatória (variabilidade de curto prazo). Essa técnica possibilitou a separação dos sinais de baixa frequência dos de alta frequência. Logo após, aplicou-se a técnica das ondeletas para se observar quais foram os períodos, ou ciclos, dominantes nas duas series criadas (longo e curto prazo) e identificar as possíveis associações entre as escalas de tempo e fenômenos que afetam o clima. No segundo momento, utilizou-se a técnica das Médias Móveis para gerar uma nova série de longo prazo com o objetivo de se fazer um comparativo com a série de tendência (sinais de baixafrequência) gerada pelo FHP. No terceiro momento, utilizou-se a técnica das ondeletas nas series do FHP e da Média Móvel para se observar os períodos de sinais, ou variações, característicos das novas series geradas pelo FHP e a pela Média Móvel e compará-los com os das séries originais. Neste estudo, optou-se pela utilização de programas gratuitos e que usam a licença General PublicLicense (GPL). Para a organização dos dados dos índices climáticos utilizou-se o programa Calc da plataforma BrOffice (LibreOffice). No cálculo do filtro Hodrick-Prescott (HP), fez-se o uso do programa estatístico $\mathrm{R}$ e o pacote "mFilter" e, para o calculo das ondeletas, foi utilizado o programa QtOctave.

\subsection{Médias móveis}

A Média Móvel (MM) é uma técnica muito utilizada pelos meteorologistas para suavizar uma série temporal, reduzindo picos instantâneos dos sinais de alta frequência existentes nas observações. Quando aplicada, a técnica elimina as 
flutuações de alta amplitude existentes na serie, criando um novo conjunto de dados e uma nova série temporal sem os "ruídos" indesejados. Existem várias formas de se calcular as médias móveis, como a aritmética e a ponderada, sendo a primeira mais utilizada comumente. Pode-se definir as médias móveis aritméticas da seguinte forma:

$$
\frac{Y_{1}+Y_{2}+\cdots+Y_{N}}{N}, \frac{Y_{2}+Y_{3}+\cdots+Y_{N+1}}{N}, \frac{Y_{3}+Y_{4}+\cdots+Y_{N+2}}{N}
$$

Isto é, uma MM aritmética de ordem $\mathrm{N}$ pode ser obtida por uma sequência de médias aritméticas. Quando a soma dos numeradores é efetuada denominam-se totais móveis de ordem $\mathrm{N}$. Se o conjunto de dados é fornecido em meses ou anos, por exemplo, as MM de ordem $\mathrm{N}$ são chamadas de médias móveis de $\mathrm{N}$ meses ou médias móveis de $\mathrm{N}$ anos. Salienta-se que a unidade de tempo pode variar conforme a necessidade do usuário. Mediante o emprego dessa técnica, com as respectivas ordens apropriadas, podem ser eliminadas as variações cíclicas, estacionais e irregulares, deixando apenas o movimento de tendência no conjunto de dados da série temporal. Lembra-se que o uso das médias MM apresenta algumas desvantagens como a perda de informações da série original.

Uma das formas mais comuns de se usar MM é a suavização de um conjunto de dados. Davis (1973) observou que, em uma série temporal, a sequência dos dados trazem alguns ruídos embutidos, isto é, observações consistem em duas partes básicas: um sinal óbvio na série (um padrão de variação que é significativo) e um ruído que se sobrepõeao sinal (uma variação aleatória). Essas expressões são mais prováveis de serem encontradas em problemas de séries temporais, como no caso das series temporais de variáveis meteorológicas. O processo de suavização também é conhecido como filtragem ea equação matemática que descreve o cálculo é chamada de filtro. Com essa noção, alguns autores consideram as MM como uma espécie de filtro que, quando aplicado, remove os ruídos (amplitudes altas do sinal) deixando apenas o sinal óbvio da serie.

A integração das MM com outras técnicas também é comum na literatura. Lucio et al. (2010) utilizaram modelos estocásticos do tipo Auto Regressivo Integrado a Médias Móveis (ARIMA) para previsão sazonal da precipitação no Brasil.

\subsection{Filtro Hodrick-Prescott}

Hodrick e Prescott (1980) propuseram um método, um filtro, que leva seu nome $(H P)$, que é uma análise estatística para tratamento de séries temporais que separa a componente da tendência da componente cíclica/aleatória existentes nos dados por meio da remoção das flutuações de baixa frequência das séries. A hipótese é que, nessa separação, a variabilidade de baixa frequência represente 
a tendência de prazo mais longo e a de frequência mais altarepresente os ciclos mais curtos e/ou aleatórios.

Em 1997, Robert Hodrick e Edward Prescott popularizaram uma técnica estatística para tratamento de séries temporais, proposta, inicialmente, por Edmund Taylor Whittaker em 1923. Tal análise é utilizada para obter uma representação suavizada e não-linear de uma série temporal, na qual, esta seria mais sensível em longo prazo do que as flutuações de curto prazo. Ou seja, obtém-se uma série de tendência não-linear suavizada. É uma técnica muito usada em ciclos reais de negócios (macroeconomia) para extrair a tendência de séries de dados, como a do produto interno bruto (PIB), por exemplo, e não tem sido aplicada a series de variáveis climáticas comumente.

O filtro Hodrick-Prescott (HP) decompõe uma série temporal em uma componente de tendências e outra cíclica/aleatória. Balcilar (2009) demonstrou a utilização do filtro $\mathrm{HP}$, considerando a decomposição de uma série temporal $x_{t}$ como a soma do período da oscilação $y_{t}$ e $o$ valor médio de $x_{t}$ para cada componente da mesma. Sendo $x_{t}$ representado por sua variabilidade no tempo, $\left\{x_{t}\right\}_{t=1}^{T}$.

$$
x_{t}=y_{t}+\overline{x_{t}}
$$

ondey varia entre $\mathrm{P}_{\mathrm{l}}$ e $\mathrm{P}_{\mathrm{u}}$, sendo que $2 \mathrm{P}_{\mathrm{l}}<\mathrm{P}_{\mathrm{u}}<\infty$. Assume-se, ainda, que $\mathrm{y}_{\mathrm{t}}$ representa uma frequência no intervalo $\{(a, b) \cup(-a,-b)\} \in(-\pi, \pi)$, podendo a e b serem escritos como:

$$
a=\frac{2 \pi}{P_{u}} e b=\frac{2 \pi}{P_{l}}
$$

Para uma quantidade infinita de dados, pode-se utilizar um filtro ideal, no qual se relacione $y_{t}$ e $x_{t}$ incluindo uma defasagem $B(L)$. Logo, $y_{t}$ pode ser escrito em função de $x_{t}$ da seguinte forma:

$$
y_{t}=B(L) \cdot x_{t} B(L)=\sum_{-\infty}^{\infty} B_{j} \cdot L^{j}, L^{k} \cdot x_{t}=x_{t-k}
$$

Ao se utilizar um filtro ideal, os pesos de $B_{j}$ são escritos em funções de "a" e " $b$ ", tendo $\mathrm{B}_{0}$ e $\mathrm{B}_{\mathrm{j}}$ definidos como:

$$
B_{0}=\frac{b-a}{\pi} B_{j}=\frac{\operatorname{sen}(j b)-\operatorname{sen}(j a)}{j \pi}
$$

O filtro HP obtém a solução de $B_{j}$ através dos pesos de $\widehat{B}_{j}$ e descritos como a solução de: 


$$
\hat{B}_{j}=\operatorname{argmin} E\left\{\left(y_{t}-\hat{y}_{t}\right)\right\}^{2}=\operatorname{argmin}\left\{\sum_{t=1}^{T}\left(y_{t}-\hat{y}_{t}\right)^{2}+\lambda \sum_{t=2}^{T-1}\left(\hat{y}_{t+1}-2 \hat{y}_{t}+\hat{y}_{t-1}\right)\right\}
$$

Para séries finitas, os valores de $\widehat{B}_{j}$ podem ser encontrados pela equação:

onde

$$
\widehat{B}_{j}=\frac{1}{2 \pi} \cdot \int \frac{4 \lambda(1-\cos (\omega))^{2}}{1+4 \lambda(1-\cos (\omega))^{2}} \cdot e^{i \omega \omega} \cdot d \omega
$$

$$
\lambda=\left(2 \cdot \operatorname{sen}\left(\frac{\pi}{\text { frequência }}\right)\right)^{-4}
$$

A frequência permitiu-nos observar a duração e a variação dos ciclos nas séries temporais, nesse caso, a variabilidade de longo prazo observada nos dados mensais dos índices climáticos. A frequência penalizou a parte da tendência interdecadal da série, separando-a da parte cíclica/aleatória (variabilidade inteannual).

Para este estudo, optou-se pelo o uso do valor da frequência de 14400 , pois, na literatura, há relatos de que esse seria o valor mais adequado para dados mensais (Ahumada e Garegnani, 1999; Balcilar, 2009). Posteriormente, serão testados valores distintos desse valor. Convém esclarecer que foram empregados valores mensais dos índices porque alguns autores sugerem que a utilização de dados anuais no filtro HP torna-se inviável pela quantidade de erros gerados devido ao pequeno comprimentodas séries temporais.

\subsection{Análise das ondeletas}

Essa técnica estatística foi se desenvolvendo ao longo dos anos e está baseada nas transformadas de Fourier (TF). Gabor (1946) estudou e adaptou a TF para o estudo do sinal no domínio do tempo e no domínio da frequência. Essa modificação ficou conhecida como TF com janela móvel. Porém, existia um grande inconveniente nessa técnica, que era o comprimento fixo que tais janelas móveis tinham que ter. Exemplificado, nos estudos em Meteorologia, os fenômenos mudam e apresentam diferenças em escalas de tempo e frequência e, muitas vezes, com ordens de grandezas diferentes para caracterizar um sinal meteorológico. Morlet et al. (1982) e Grossman \& Morlet (1984) analisaram o estudo feito por Gabor e o modificaram, introduzindo a Análise de Ondeletas (AO). A AO satisfaz o Princípio de Heisenberg, introduzindo janelas que se adaptam conforme a frequência dos fenômenos que ocorrem, ou seja, essas janelas adaptam-se em relação a fenômenos de baixa ou alta frequência.

Weng \& Lau (1994) descreveram a AO como uma série de funções em pequenas ondas. Essas têm sua gênese através de alargamento e translações de uma função simples de variável real qualquer e são, também, conhecidas como 
ondeleta-mãe. Tal função pode ser descrita matematicamente tomando-se uma escala e uma posição, onde o valor da escala tem que assumir valores maiores que zero. A equação a seguir mostra função da transformada de ondeletas (TO) em uma série continua:

$$
\left(W_{\Psi} f\right)(a, b)=\frac{1}{\sqrt{a}} \int f(t) \Psi\left(\frac{t-b}{a}\right) d t
$$

onde $f(t)$ é a função que constitui a série de dados a ser analisada, $\frac{1}{\sqrt{a}}$ é utilizado para normalizar a energia de cada ondeleta; "a" é a escala, "b" é a posição e " $t$ " é a variável real.

Na literatura, existem várias funções que são utilizadas para o cálculo e geração das ondeletas (DAUBECHIES, 1992; FOUFOULA-GEORGIOU E KUMAR, 1994). Mas, as que são mais utilizadas são as funções de Paul, Dog e Morlet. Cada uma possui sua peculiaridade e a função de Morlet foi utilizada neste estudo. Essa foi escolhida, pois, segundo Weng \& Lau (1994), é uma função complexa, que possui características semelhantes às dos sinais meteorológicos que se desejam analisar, tais como simetria, ou não, e apresenta uma variação temporal suave ou brusca. A função de Morlet é descrita pela seguinte equação:

$$
\Psi(t)=\pi^{\frac{-1}{4}} \cdot e^{i W_{0} t} \cdot e^{\frac{-t^{2}}{2}}
$$

onde " $W_{0}$ " é a frequência adimensional de Morlet e " $t$ " é o parâmetro adimensional no tempo.

A TO consiste, apenas, em decompor um sinal em diferentes partes, ou seja, explora-se tal sinal em seus níveis de resolução. Esse procedimento é conhecido na literatura como multirresolução. Ao ser apresentada, a multirresolução mostra uma figura simplificada para a análise da informação que o sinal trás. As várias resoluções, ou seja, os vários detalhes de um sinal, de forma geral, caracterizam as diferentes estruturas físicas que estão contidas no mesmo. Então, ao modular essa resolução, pode-se trazer à tona sinais mais grosseiros ou mais refinados presentes nas séries temporais. Esses sinais são apresentados na forma da intensidade de energia que se representa em função da frequência (período) e da escala temporal da série.

\section{Resultados e discussão}

3.1. Componentes tendência e cíclica/aleatória da série dos índicesda ODP resultantes da aplicação das técnicas Médias Móveis e Filtro HodrickPrescott

Na Figura 1 retratou-se a série dos valores mensais dos índices da Oscilação Decadal Pacífico (ODP) para o período de janeiro de 1900 a dezembro de 2011 e as fases da ODP bem definidas, como descrito na literatura, uma fase fria de 
1947 a 1976 e uma fase quente de 1977 a 1998. Notou-se que a série parece dar viradas climáticasbruscas na passagem de uma fase para outra, que podem estar associados a mudanças bruscas do armazenamento de calor no oceano e, consequentemente, da temperatura da superfície do mar (TSM). Molion e Lucio (2012) sugeriram que a ODP não seja uma oscilação regular e que essas viradas estejam associadas a ondas internas provocadas por terremotos de grande magnitude, como o das Ilhas Aleutas (região de águas frias) em abril de 1946, que coincidiu com o início da fase fria da ODP e o de Tangshan (China) em julho de 1976, que afetou a chamada piscina de águas quentes e que coincidiu com o início da fase quente da ODP. No período de 1946 a 1976, a região centro equatorial do Pacífico ficou mais fria que a média do período 1946-2011, ou seja, índices oudesvios de TSM, negativos na maior parte do período. Na fase quente, ocorreu o oposto.Dentro de cada fase, houve anos em que os índices alternaram seu sinal. Por exemplo, na fase fria, o forte evento El Niño de 1957 a 1962 fez com que os índices da ODP apresentassem valores positivos.

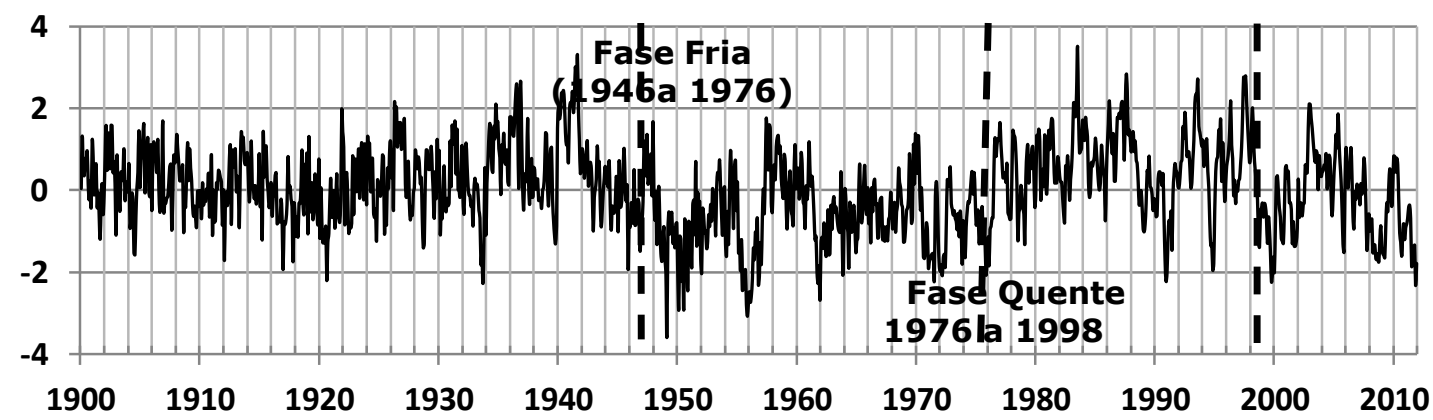

Figura 1: Índices mensais da Oscilação Decadal do Pacífico (ODP) de janeiro de 1900 até dezembro de 2012. Fonte dos dados: ESRL/PSD/NOAA, JISAO.

Molion (2005) mostrou que houve uma inesperada coincidência entre o comportamento da temperatura do ar média global (Ta) e a ODP, com a Ta diminuindo entre os anos de 1948 - 1976 (fase fria) e aumentando durante 1977 - 1998 (fase quente). Também notou que durante a fase fria da ODP os eventos La Niña foram mais intensos e prolongados e os eventos El Niño foram, em sua maioria, menos intensos e curtos. Na fase quente da ODP, ocorreu o inverso. Siqueira et al. (2006) estudaram a relação entre a ODP e os impactos causados sobre o Norte e Nordeste Brasileiro e mostraram que, dependendo da fase da ODP, os ENOS causam impactos diferentes. Siqueira et al. (2007), também, elaboraram um estudo sobre a variabilidade climática no sudeste da Amazônia, fronteira de expansão da soja no Brasil. Constataram que no período de 1948 - 1976, embora tenha sido um período de predominância de eventos La Niña, houve uma diminuição dos totais pluviométricos sobre a região e, durante 1977 - 1998, ocorreu o contrário. Concluíram que essa variabilidade pode ter impactos negativos na safra dessa commodity se a ODP permanecer em sua nova fase fria nos próximos 20 anos. 
Siqueira e Molion (2008), por sua vez, mostraram que durante os eventos La Niña da fase fria da ODP, os totais pluviométricos sobre as Regiões Norte e Nordeste do Brasil foram abaixo da normal climatológica em relação à média do período que compreende as duas fases da ODP (1948 - 1998), ao contrário do que consta na literatura. Ou seja, a afirmação que eventos La Niña causam desvios positivos de precipitação nessas regiões só foi verdadeira para a fase quente da ODP. Os eventos El Niño se apresentam de forma semelhante nas duas fases da ODP, causando desvios negativos de precipitação sobre essas regiões, sendo mais severos os da fase quente. Muito pouco tem sido relatado sobre a importância, impactos e influência da ODP no clima global. No entanto, devido ao fato de os oceanos ocuparem $70 \%$ da superfície do planeta, e o Oceano Pacífico representar a metade dos oceanos, a ODP deve certamente ter influência na variabilidade climática de prazo longo, a ponto de ser confundida com mudanças climáticas.

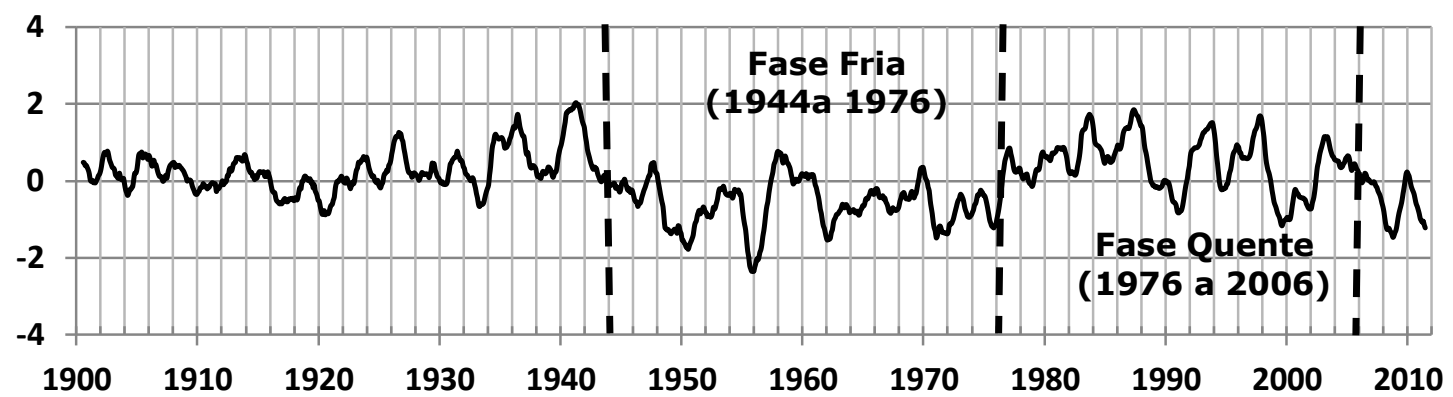

(a)

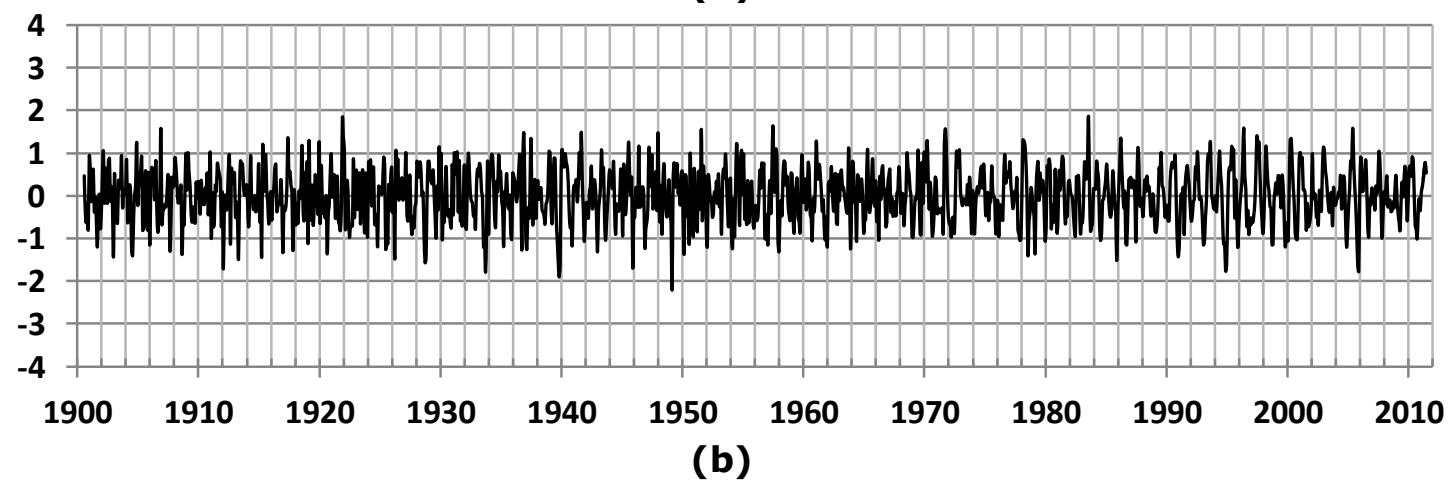

Figura 2: (a)Tendência (variabilidade de longo prazo) extraída aplicando MM de 13 meses e (b) parte cíclica (variabilidade de curto prazo) extraída com a subtração da série dos índices da ODP da série de tendência.

$\mathrm{Na}$ Figura 2a, apresentou-se a tendência do índice da ODP, extraída com a aplicação da técnica das MM de 13 meses, em que a variabilidade de longo prazo é aparente. Contudo, a suavização mostrou que a curva da tendência (baixa frequência) teve a interferência da variabilidade de curto (alta frequência), talvez por filtrar os ciclos anual e intrasazonal apenas. Ou seja, se o evento for de duração superior a 1 ano, como é o caso de alguns eventos ENOS, seu sinal se torna bem aparente na curva de tendência, pois não foi filtrado. 
Notou-se que a fase fria da ODP parece começar em 1944, 3 anos antes do descrito na literatura (Figura 1). A fase quente teve inicio, em ambos os casos, entre os anos de 1976 e 1977, masseu término não coincide. Na Figura 1, o fim da fase quente foi observado no ano 1998 e, na Figura 2a, no ano de 2006. Semelhante ao observado na série original, durante a fase fria, o forte evento El Niño de 1957 a 1962 fez com que os índices da ODP apresentassem valores positivos atípicos. Na fase quente da ODP,o comportamentofoi o mesmo visto na Figura 1. O forte evento La Niña de 1988/90 e, possivelmente, a erupção do Monte Pinatubo, em junho de 1991, tenham contribuído para geraríndices negativos temporariamente, retornando a índices positivos em seguida. A técnica de MM tem um inconveniente que é a perda de dados no início e no final da série, que é indesejável quando a série não é suficientemente longa e o valor selecionado para o intervalo do filtro é elevado.

$\mathrm{Na}$ Figura 2b, a parte cíclica/aleatória (variabilidade de curto prazo),extraída pela subtração da série de tendência da série original, notou-se que as amplitudes do sinal são pequenas e a série de resíduos gerada é composta basicamente dos ciclos anual, sazonal e intra-sazonal.
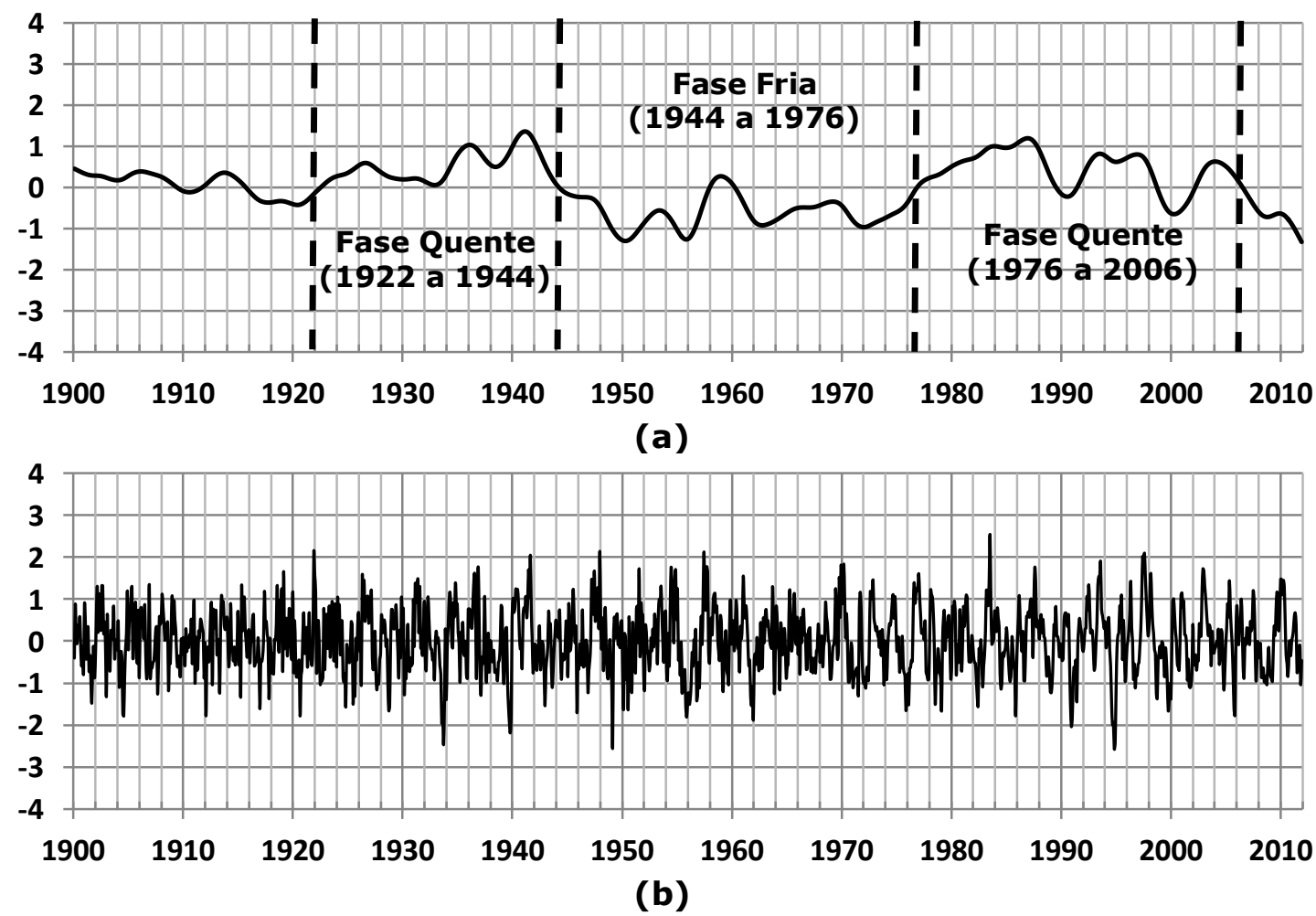

Figura 3:(a) Tendência de longo prazo (variabilidade de baixa frequência) e (b) parte cíclica/aleatória (variabilidade de alta frequência) da série dos índices da ODP extraída com aplicação do Filtro Hodrick-Prescott (FHP).

$\mathrm{Na}$ Figura 3a, a curva das tendências evidenciou as oscilações de baixa frequência, ou seja, variabilidade de longo prazo, interdecadal possivelmente associada à ODP, com uma fase quente entre 1922 e 1944, seguida de uma fase 
fria entre 1944 e 1976. Mantual et al (1997) sugeriram que essa fase fria tenhacomeçado em 1946 - 1947. Outros aspectos seriama extensão e o término da fase quente recente da ODP (período de 1976 a 1998) que, segundo estaanálise, não teria sido em 1998 e sim em 2006, dando inicio a nova fase fria da ODP após essa data. Essa divergência pode ter ocorrido devido ao forte La Niña de 1999 a 2002, que foi substituído pelos eventos El Niño de 2002/03 e 2004/05. A partir de 2006, os índices se tornaram negativos. De maneira geral, a curva de tendências apresentou valores negativos na fase fria da ODP. Em alguns pontos, porém, foram notadas inversões bruscas do comportamento da serie. Na fase fria da ODP, entre 1957 e 1960, notou-se, claramente, uma inversão, com um pico em 1958, concordando com a análise anterior (Figura 2a). O declínio dos índices no período compreendido entre 1988 e 1990 também foi observado, porém com intensidade menor.

Na Figura 3b, foram apresentadas as altas frequências (variabilidade de curto prazo), a parte cíclica/aleatória do filtro HP. As amplitudesdo sinal são maiores que suas respectivas obtidas com a aplicação das MM (Figura 2b), evidentes, por exemplo, nos eventos fortes de El Niño de 1982/83 e 1997/98. Ou seja, aparentemente a parte cíclica/aleatória do filtro HP tende a ressaltar eventos extremos de escala interanual, como os eventos ENOS fortes, que tenham frequências intermediárias. Para estudos de clima, o filtro HP parece ser uma técnica mais adequada, pois sua curva de tendência é mais suave,indicando que frequências altas são filtradas mais eficientemente, sem perda de dados e apresentam um sinal com mais energia em sua parte cíclica/aleatória.

\subsection{Análises das ondeletas das séries original e geradas pelas técnicas de filtragem}

Nas Figuras 4 e 5 mostraram-se os resultados da análise das ondeletas para o índice da ODP, tendência e parte cíclica extraídas com aplicação das técnicas Médias Móveis e filtro Hodrick-Prescott, respectivamente, com o Espectro de Potência (EPO) à direita e o Espectro Global (EGO) à esquerda.

No EPO, as curvas fechadas, são mostradas as variâncias normalizadas variando de 0 a 5 unidades com o intervalo de contorno de 0,5. Os contornos fechados realçados são significativos ao nível de confiança superior a $95 \%$ e a curva tracejada, em forma parecida à letra " $U$ " alongado, representa o cone de influência, sob o qual o efeito de borda se faz importante. NoEGO, os picos, que ultrapassam a curva negra tracejada à direita, indicam as periodicidades do sinal, ou ciclos aparentes nas séries, possivelmente associados a fenômenos com ciclos conhecidos, como manchas solares e ciclo nodal lunar. 


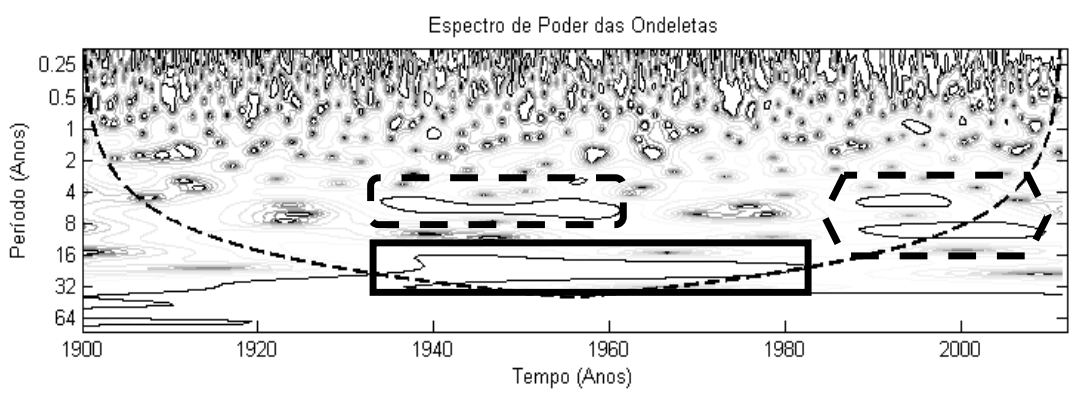

(a)

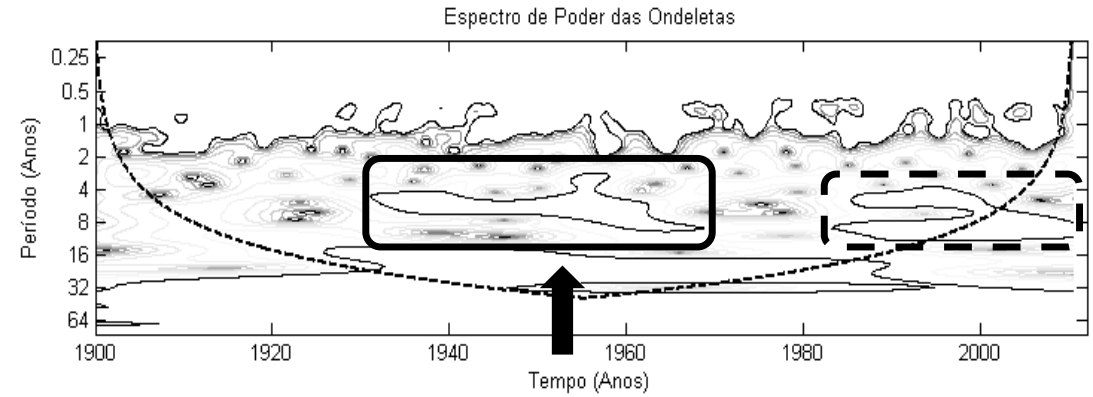

(b)
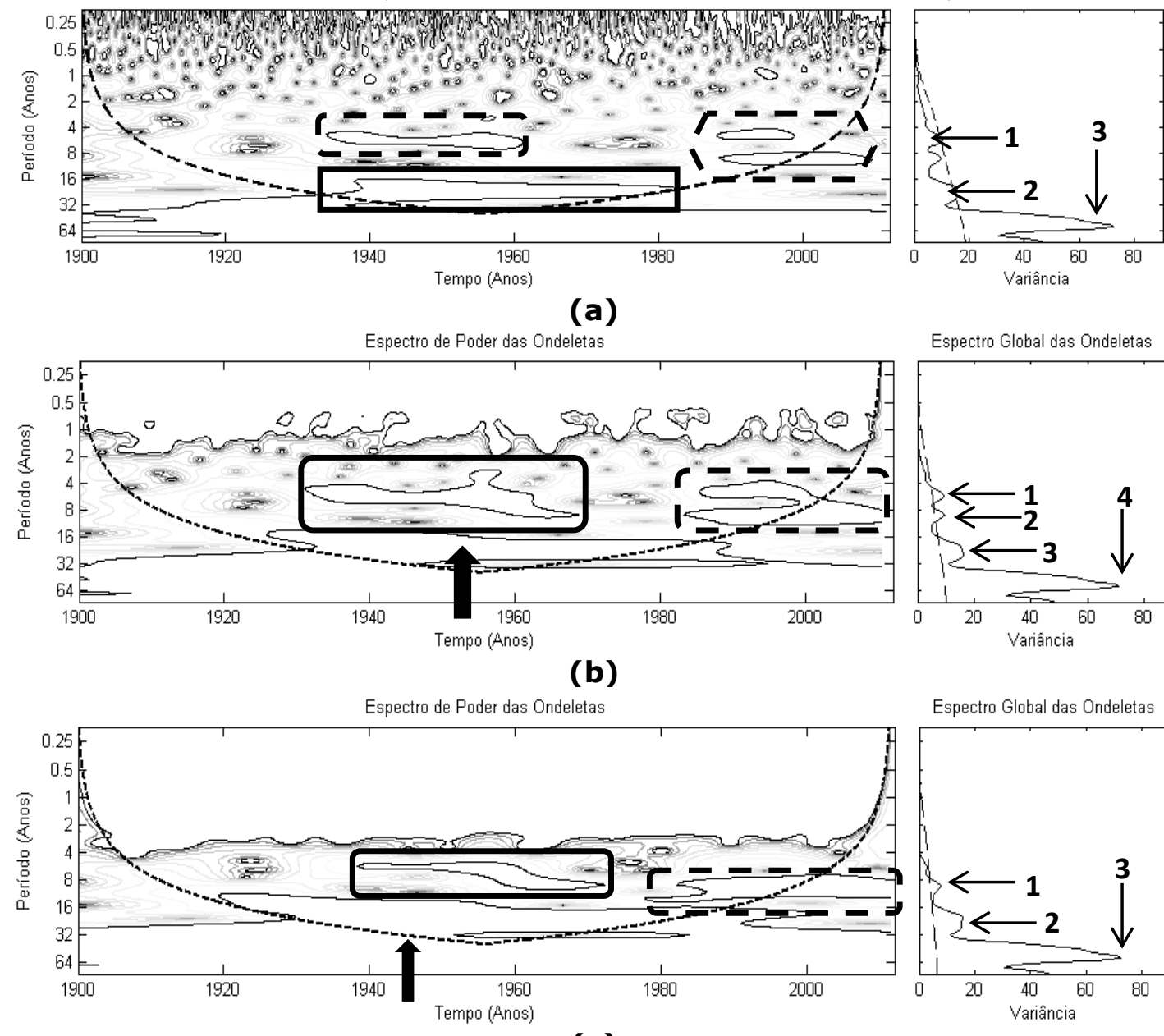

Espectro Global das Ondeletas

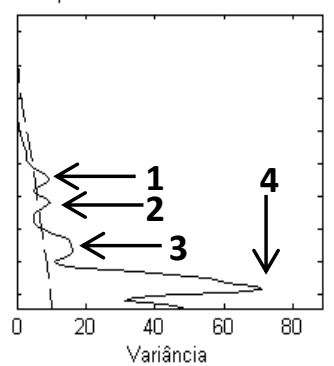

Espectro Global das Ondeletas

(c)

Figura 4: Analise das ondeletas aplicada à série dos (a) índices da ODP, séries das tendências obtidas com aplicação de (b) MM e (c) Filtro HP, com respectivos Espectros de Potência (EPO, esquerda) e Espectro Global (EGO, direita).

No EPO da série dos índices da ODP (Figura 4a) notaram-se núcleos significativos que apresentaram uma alta energia nos intervalos dos períodos de 2 a 8 anos (retângulo negro tracejado) entre os anos de 1930 e 1960, de 4 a 8 anos e de 8 a 16 anos (hexágono negro tracejado) entre os anos de 1980 e 2008 e, de 16 a 32 anos (retângulo negro) entre os anos de 1930 e 1982. No EGO, observaram-se picos dominantes que apresentaram grande variância nas escalas de 4 a 8 anos (seta 1), 16 a 32 anos (seta 2) e 32 a 64 anos (seta 3). A ODP apresentou uma mudança de fase em entre 1946 e 1947. Observou-se que o primeiro intervalode alta energia no EPO (retângulo negro tracejado) localizou-se justamente nesse período de transição entre a fase quente (1925 a 1946) e fria (1947 a 1976).Comparando a Figura 4acom o Índice da Oscilação Sul (não mostrado), notou-se que esse período foi marcado comuma grande variação entre eventos El Niño (de 1930 a 1946) e eventos La Niña (de 1947 a 1960). Os ciclos de 4 a 8 anos (variabilidade interanual), evidentes no EGO 
(seta 1), possivelmente estão associados à alta repetitividade e intensidade dos eventos ENOS.

O segundo período (hexágono negro tracejado), também foi detransição entre as fases da ODP. Porém, essa transição também ocorreu no final da fase quente (1976 a 1998) para o início da nova fase fria (a partir de 1999). Os ciclos também foram com intervalo de tempo de 4 a 8 anos, semelhantes ao do período 1930-1982. Também, observou-se um núcleo de ciclo de 8 a 16 anos durante o mesmo período, provavelmente associado à variabilidade da ODP e da OMA (Oscilação Multidecadal do Atlântico), pois, nesse mesmoperíodo, a ODP estava em sua fase positiva e a OMA em seu modo negativo. Aparentemente, a oposição de fases tende a mudar a circulação da atmosfera globalmente e interferir na frequência e intensidade dos eventos ENOS. No terceiro período (retângulo negro), observou-se uma variação entre 16 e 32anos, evidenciada pelo segundo pico a ultrapassar a linha designificância (seta 2) no EGO. Tal resultado indica uma variabilidade decadal, como a ODP, que pode estar associada ao ciclo solar de 22 anos, por exemplo. Observou-se, ainda, que o EGO apresentou uma grande variância no intervalo de 32 a 64 anos, que ultrapassou a linha de significância (seta 3), indicandouma variabilidade correspondente aum ciclo completo da ODP, fase quente mais fase fria.Contudo, essa afirmação deve ser tomada com cautela, pois a série dos índices de ODP é relativamente curta, uma vez que é necessário um intervalo de observações de, no mínimo, duasvezes o ciclo do fenômeno a ser detectado.No caso, o intervalo de observações deveria ser superior a 120 anos.

Ainda na mesma figura foram mostradas as análises de ondeletas para a componente da tendência da série dos índices da ODP extraída com aplicação das MM em (Figura 4b) e com o filtro HP em (Figura 4c). Observaram-se, no EPO, em ambas as análises, picos de energias parecidos, porém deslocados em tempo. No EGO, notaram-se 4 picos significativos na análise da tendência extraída com as MM e 3 picos significativos na análise feita com a tendência extraída com o filtro HP. Em comparação com a Figura 4a, observou-se que as tendências das séries filtradas ressaltaram os 3 picos de energia associados à variabilidade interdecadal de 8-16, 16-32 e 32-64 anos. O pico de 4-8 anos (seta 1, Figura 4b) não apareceu no EGO da tendência com o filtro HP porque os fenômenos de escala interanual foram devidamente filtrados e sua energia transferida para a parte cíclica/aleatória (Figura 4c). No ciclo 8-16 anos do EGO, o pico do sinal está em 9 anos, o que sugere uma associação com um submúltiplo do ciclo nodal lunar de 18,6 anos (9,3 anos).

$\mathrm{Na}$ Figura 5foram mostradas as análises com as ondeletas da componentecíclica/aleatória (variabilidade de curto prazo) da série dos índices da ODP com aplicação de (a) Médias Móveis e (b) filtro HP. 

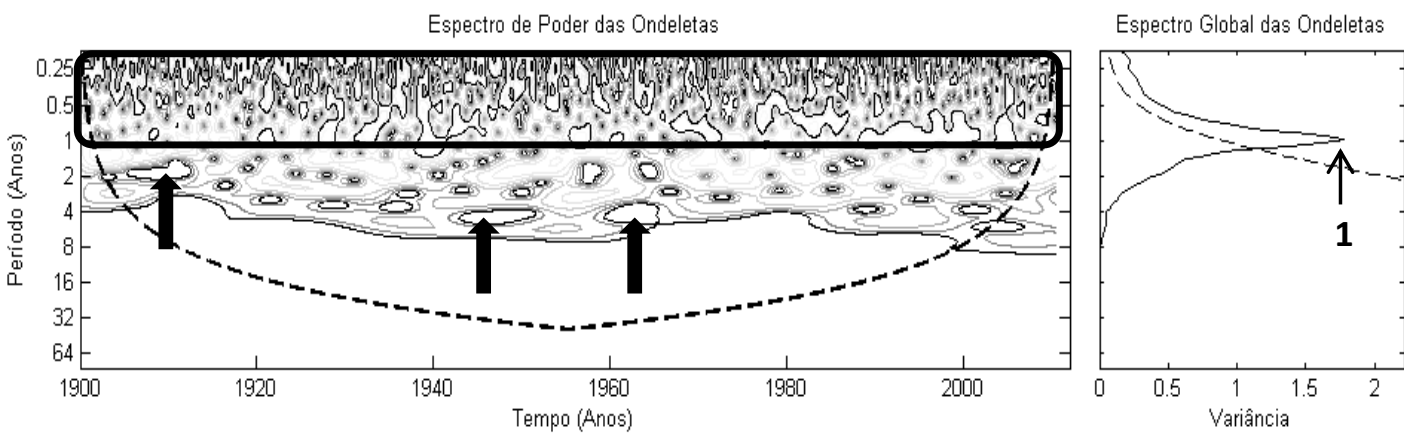

(a)
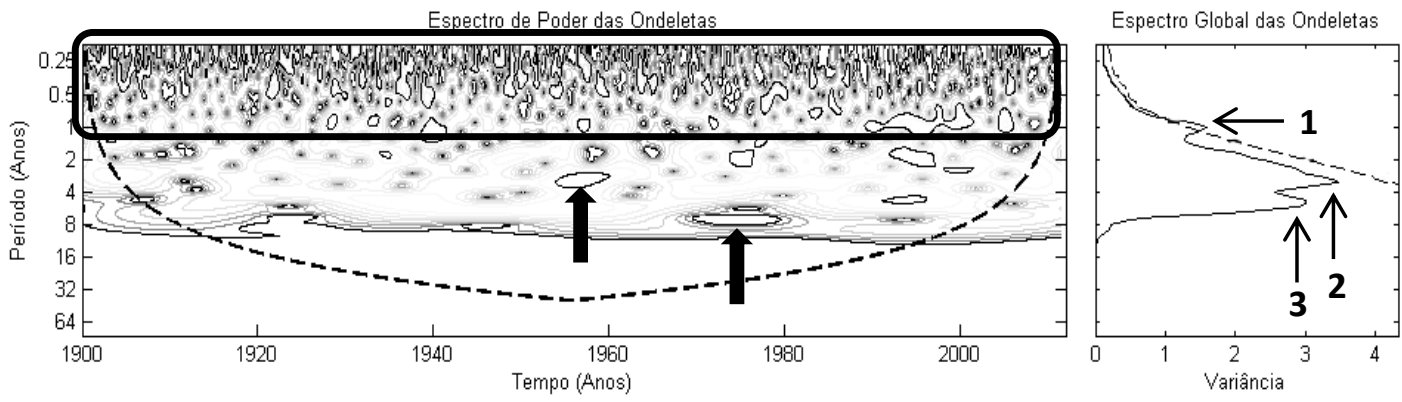

(b)

Figura 5: Analise das ondeletas aplicada às series da componente cíclica/aleatória obtidas com aplicação de (a) Médias Móveis e (b)com filtro HP, com respectivos Espectros de Potencias (EPO, esquerda) e Espectro Global das Ondeletas (EGO, direita).

Os resultados mostraram o ciclo anual claramente e as variabilidade sazonal e intra-sazonal nos dois EGO. Como era de se esperar, o EGO obtido com o filtro HP (Figura 5b) mostrou os ciclos de 2-4 e 4-8 anos a um nível de significância um pouco inferior a $95 \%$ que não foram mostrados no EGO obtido com as MM (Figura 5a). Ou seja, a técnica das MM, utilizando intervalo de 13 meses como parâmetro do filtro, dá ênfase aos ciclos anual, sazonal e intrasazonal e removeos sinais dos eventos interanuais (de 2 a 10 anos), ou seja variabilidade inferior à decadal.Esses sinais filtrados passam a compor a série da tendência que fica mais ruidosa e não representa realmente a variabilidade de longo prazo interdecadal (baixas frequências), a climática propriamente dita. Comparandose as Figuras 2 e 3, vê-se claramente, que a curva da tendência obtida com as $\mathrm{MM}$, por conter a variabilidade inferior à decadal, é mais ruidosa (Figura 2a) e a do filtro HP mais suave (Figura 3a), enquanto o sinal da componente cíclica/aleatória obtida com as MM (Figura 2b) apresenta uma amplitude inferior àquela obtida com o filtro HP (Figura $3 b$ ), que contém a variabilidade inferior à decadal.

\section{CONCLUSÕES}

O filtro Hodrick-Prescott comprovou ser uma ferramenta mais adequada para a análise de séries temporais de variáveis meteorológicas em estudos de clima, separando as componentes referentes à tendência e àcíclica/aleatória das 
séries.As frequências altas dos eventos interanuais (2 a 10 anos) são removidas eficientemente da série original e são incorporadas na curva cíclica/aleatória como mostrou a análise das ondeletas. Isso gerauma curva de tendência mais suave que contém frequências baixas apenas e ressalta a variabilidade climática de longo prazo, que é um objetivo dos estudos climáticos. A técnica das Médias Móveis com parâmetro de filtro de 13 meses, ao contrário, não remove as frequências dos eventos interanuaisda série original, sendo estas incorporadas na curva de tendência, que se torna ruidosa e dificulta a interpretação dos resultados das análises climáticas. A técnica das $M M$ ainda possui o inconveniente da perda de dados no início e no final da série,que compromete o uso de seus resultados no prognóstico climático se o parâmetro de intervalo do filtro for muito elevado e a série relativamente curta. De maneira geral, a interpretação física dos resultados das análises estatísticas confirmou os já encontrados por outros pesquisadores, como o ciclo longo de 50-60 anos da ODP, e os de frequência mais alta, de 3 a 7 anos, associados aos eventos ENOS que são interanuais. Concluiu-se que o filtro HP é uma técnica estatística mais adequadaque a das Médias Móveis paraanálise de series temporais de dados meteorológicos, em que a tendência climática de prazo longo é o foco principal.

Agradecimentos: $O$ primeiro autor agradece a FINEP e ao CNPQ pela concessão da bolsa DTI.

\section{REFERÊNCIAS}

AHUMADA, H. \& GAREGNANI, L. Hodrik-Prescott filter in practice. Económica, Departamento de Economía, Facultad de Ciencias Econòmicas, Universidad Nacional de La Plata, vol. 0(4), pages 61-76.

BALCILAR, M. Miscellaneous time series filters, mfilter package. $R$ Statistical Software, 26 p., 2009.

DAUBECHIES, I.: Ten Lectures on Wavelets.SIAM, 357p, 1992.

DAVIS, J.C. Statistics and Data Analysis in Geology.Wiley International Edition, John Wiley \& Sons, Inc., New York, 550 pp. - 1973

FOUFOULA-GEORGIOU, E.; KUMAR, P.: Wavelets in Geophysics: wavelet analysis and its applications. San Diego, Academic Press, 1994, 372p.

GABOR, D.: Theory of communication. J.Inst.Elect.Eng., 93, 429-457, 1946.

GROSSMAN, A; MORLET, J. 1984. Decomposition of hardy functions into square integrable wavelets of constant shape. Society for Industrial and Applied Mathematics Journal on Mathematical Analysis, 15:732-736.

HODRICK, R. J. AND PRESCOTT, E.C.Postwar U.S. Business cycles: an empirical investigation; mss. Pittsburgh: Carnegie-Mellon University; Discussion Papers 451, Northwestern University, 1980. 
LUCIO, P.S. et al. Um modelo estocástico combinado de previsão sazonal para a precipitação no Brasil. Revista Brasileira de Meteorologia, v.25, n.1, $70-87,2010$.

MANTUA, N. J. et al (1997). "A Pacific interdecadal climate oscillation with impacts on salmon production". Bulletin of the American Meteorological Society 78 (6): 1069-1079. Bibcode 1997BAMS...78.1069M.doi:10.1175/15200477(1997)078<1069:APICOW > 2.0.CO;2.

MOLION, L.C.B. Aquecimento global, El Niños, manchas solares, vulcões e Oscilação Decadal do Pacífico,Climanalise, agosto, CPTEC/INPE, 2005.

Disponível em:

http://www.cptec.inpe.br/products/climanalise/artigos/molion0805.pdf.

Acessado em 10 de dezembro de 2010 às 20 horas.

MOLION, L.C.B. e LUCIO, P.S. Note on the Pacific Decadal Oscillation, EI Niño-Southern Oscillation and Intertropical Front in Sahel, Africa, accepted for publication in Atmospheric and Climate Sciences.

http://www.scirp.org/journal/acs/, 2012.

MORLET, G. A.; FOURGEAU, I.; GIARD, D. Wave propagation and sampling theory. Part 1: complex signal and scattering in multilayered media. Geophysics, v. 47, n 2, p. 203-221, 1982 a.

. Wave propagation and sampling theory. Part 2: sampling theory and complex waves. Geophysics, v. 47, n. 2; p. 222-236, 1982b.

NOBRE, C. A.; MOLION, L. C. B. The climatology of droughts and drought prediction. In: The impact of climatic variations on agriculture, Ed. M. L. Parry, T. R. Carter and N. T. Konijin, IIASA, Kluver Academic Pub., p. 305-323, 1988.

RASMUSSON, E. M.; WALLACE J. M. Meteorological aspects of the El NiñoSouthern Oscillation. Science, v. 222, p. 1195-1202, 1983.

ROPELEWSKY, C. F. e HALPERT, M. S. (1987) Global and regional scale precipitation patterns associated with the EI Niño-Southern Oscillation (ENSO). MonthlyWeatherReview, 115: 1606-1626.

SIQUEIRA, A.H.B, et al. Impactos do ENOS no Norte e Nordeste Brasileiros e sua relação com a Oscilação Decadal do Pacifico, XIV Congresso Brasileiro de Meteorologia, 2006, Florianópolis. Anais - XIV Congresso Brasileiro de Meteorologia, 2006.

SIQUEIRA, A. H. B.; MOLION, L. C. B.; SOUZA, L. O.; VAZ, J. C. M. Impactos da variabilidade climática no cultivo de soja no leste da Amazônia. In: XV Congresso Brasileiro de Agrometeorologia, 2007, Aracajú. Anais - XV Congresso Brasileiro de Agrometeorologia, 2007.

SIQUEIRA, A.H.B.; MOLION, L. C. B. El Niño-Oscilação Sul e a Oscilação Decadal do Pacífico. IX Simpósio de Recursos Hídricos do Nordeste, 2008, Salvador. Anais - IX Simpósio de Recursos Hídricos do Nordeste, 2008.

Acadêmico da UFAL, 2008.

O Atlântico Norte e os climas brasileiros. Congresso 
SIQUEIRA, Anderlan H. B. Clima da América do Sul e sua relação com os oceanos adjacentes. Trabalho de Conclusão de Curso em Meteorologia. Instituto de Ciências Atmosféricas, Universidade Federal de Alagoas. 101 f. Janeiro, 2010. Orientador: Luiz Carlos Baldicero Molion, PhD.

SPIEGEL, M. R. (1969). Estatística: resumo da teoria. COLEÇÃO SCHAUM. Traduzido por Pedro Cosentino. Ao Livro Técnico S. A. 579 p. Rio de Janeiro RJ.

WENG, H.; LAU, K.M.: Wavelet, period doubling, and time-frequency localization with application to organization of convection over the tropical western pacific. J.Atmos.Sci., 51, 2523-2541, 1994. 